\title{
Armazenamento refrigerado de pimenta dedo-de-moça 'BRS Mari’ em embalagem polimérica
}

\author{
Cold storage of “dedo-de-moça” 'BRS Mari' pepper in polymeric packaging
}

\section{Cristina Soethe $^{1 *}$, Leonora Mansur Mattos ${ }^{2}$ e Cristiano André Steffens ${ }^{1}$}

Recebido em 07/11/2016 / Aceito em 19/01/2017

\section{RESUMO}

O objetivo deste trabalho foi avaliar se há diferença entre as embalagens de polietileno tereftalato e de policloreto de vinila, bem como o efeito do tempo de armazenamento sobre a manutenção das características físico-químicas, do teor de compostos bioativos e da atividade antioxidante de pimentas dedo-de-moça 'BRS Mari' mantidas sob refrigeração. As pimentas foram colhidas aos 80 dias após a antese (DAA). Os tratamentos avaliados foram embalagens PET e de PVC e seis tempos de armazenamento $(0,3,6,9,12$ e 15 dias). Em cada embalagem foram acondicionados 30 frutos. Os frutos foram armazenados a $10 \pm 1{ }^{\circ} \mathrm{C}$ e, logo após cada tempo de armazenamento, analisados quanto à coloração (ângulo hue, luminosidade e croma), teor de sólidos solúveis (SS), acidez titulável (AT), compostos fenólicos totais, atividade antioxidante e capsantina. Não foi observada diferença entre as embalagens avaliadas para as variáveis analisadas. O tempo de armazenamento não apresentou efeito sobre o teor de SS, AT, teor de capsantina e na relação SS/AT. A luminosidade diminuiu e os valores de ângulo hue e croma aumentaram no terceiro dia de armazenamento. Teor de compostos fenólicos e atividade antioxidante diminuíram no terceiro dia de armazenamento. Não houve diferença entre as embalagens PVC e PET sobre a manutenção da qualidade e das propriedades funcionais das pimentas. O tempo de armazenamento refrigerado não alterou as características de sabor, porém já no terceiro dia de armazenamento as pimentas apresentaram comprometimento da aparência dos frutos e redução na composição de compostos fenólicos totais e na atividade antioxidante.

PALAVRAS-CHAVE: Capsicum baccatum var. pendulum, pós-colheita, qualidade, compostos bioativos, atividade antioxidante.

\section{ABSTRACT}

The objective of this work was to evaluate if there is difference between polyethylene terephthalate (PET) and polyvinyl chloride (PVC) packaging on the quality, maintenance and the effect of storage time on the maintenance of the physical and chemical characteristics, the content of bioactive compounds and antioxidant activity in cold stored 'BRS Mari' hot pepper. Fruits were harvested at 80 days after anthesis (DAA). The treatments evaluated were PVC and PET packing combined with six storage times $(0,3,6$, 9, 12 and 15 days). 30 peppers were placed in each package. Fruits were stored at $10 \pm 1{ }^{\circ} \mathrm{C}$ and, after each storage time, analyzed for color $\left(h^{\circ}\right.$, brightness and chroma), antioxidant activity and soluble solids (SS), titratable acidity (TA), SS/TA ratio, total phenolic and capsanthin. There was no substantial difference between the evaluated packages for the variables analyzed. The storage time had no effect on the soluble solids content, titratable acidity, SS/TA ratio and capsanthin content. The brightness decreased and the value of the hue angle and chroma increased on the third day of storage. The total phenolic content and antioxidant activity decreased on the third day of storage. There were no differences between the PVC and PET packaging on the maintenance of quality and functional properties. The cold storage did not change the taste characteristics, but on the third day of storage the 'BRS Mari' hot peppers showed impaired fruit appearance and reduction of total phenolic content and antioxidant activity.

KEYWORDS: Capsicum baccatum var. pendulum, postharvest, quality, bioactive compounds, atioxidant activity.

${ }^{1}$ Universidade do Estado de Santa Catarina, Lages, SC, Brasil.

${ }^{2}$ Empresa Brasileira de Pesquisa Agropecuária, Brasília, DF, Brasil.

*Autor para correspondência < crisoethe@hotmail.com> 


\section{INTRODUÇÃO}

As pimentas são excelentes fontes de antioxidantes naturais, como as vitaminas $\mathrm{C}$ e $\mathrm{E}$, capsaicinoides, carotenoides e compostos fenólicos (VERA-GUZMÁN et al. 2011, PINTO et al. 2013). Devido às suas qualidades organolépticas, como cor, sabor e aroma, é utilizada na culinária como tempero e, constitui matéria-prima para extração de corantes, aromatizantes e oleoresinas, substâncias utilizadas nos alimentos para aumentar a estabilidade oxidativa dos lipídeos (PINTO et al. 2013).

Os antioxidantes proporcionam benefícios à saúde, devido suas funções de proteção contra várias doenças, tais como câncer, anemia, diabetes e doenças cardiovasculares (EDUSEI \& OFOSU-ANIM 2013). Todavia, os níveis dos compostos antioxidantes em pimentas podem variar de acordo com a cultivar, condições de cultivo, estádio de maturação (RODRIGUEZ-MATURINO et al. 2012), manuseio pós-colheita e condições de armazenamento (TOPUZ et al. 2011).

As condições de armazenamento têm importante papel na manutenção da qualidade de frutas e hortaliças. Contudo, o metabolismo celular continua durante o armazenamento, e pode influenciar a aparência, textura, sabor e o valor nutricional do produto (EDUSEI \& OFOSU-ANIM 2013). Por essa razão o emprego da refrigeração e do acondicionamento dos frutos em embalagens poliméricas são técnicas que apresentam importante efeito no aumento da vida pós-colheita de vegetais, diminuindo os processos fisiológicos.

O armazenamento refrigerado é um dos métodos mais utilizados para conservação de frutas e hortaliças, pois consiste na redução da temperatura e no controle da umidade relativa, diminuindo o metabolismo celular e retardando a deterioração (BRACKMANN et al. 2008). Todavia, para uma melhor manutenção da qualidade organoléptica de pimentas se faz necessário o uso de embalagens poliméricas, especialmente em longos períodos de armazenamento, por reduzir substancialmente a perda de massa (MANOLOPOULOU et al. 2010) e manter as propriedades funcionais das pimentas (UTTO et al. 2013). As embalagens poliméricas possuem propriedade de barreira ao vapor d'água e aos gases $\mathrm{O}_{2}$ e $\mathrm{CO}_{2}$, reduzindo a perda de matéria fresca e mantendo a coloração das pimentas e do pedúnculo por um período maior durante o armazenamento, principalmente quando mantidas sob refrigeração (HENZ \& MORETTI 2008).

Diversas são as embalagens poliméricas disponíveis para o acondicionamento de frutas e hortaliças. Dentre as embalagens poliméricas disponíveis para o acondicionamento de pimentas, destacam-se o polietileno tereftalato (PET) e o policloreto de vinila (PVC). O PET tem sido um dos polímeros mais utilizados, devido as suas características físico-mecânicas, tais como, rigidez, resistência a umidade, boa resistência química e estabilidade à deformação (NASSER et al. 2005). Os filmes de PVC além do baixo custo, são flexíveis, o qual facilita seu manuseio e aplicação (SARANTÓPOULOS et al. 2003).

O objetivo deste trabalho foi avaliar se há diferença entre as embalagens PET e PVC sobre a conservação de pimentas dedo-de-moça 'BRS Mari', bem como o efeito do tempo de armazenamento sobre a manutenção das características físico-químicas, do teor de compostos bioativos e da atividade antioxidante dos frutos mantidos sob refrigeração.

\section{MATERIAL E MÉTODOS}

Pimentas dedo-de-moça 'BRS Mari' foram cultivadas no campo experimental da Embrapa Hortaliças, Brasília, DF. Foram utilizados frutos colhidos com 80 dias após a antese (DAA). A colheita dos frutos foi realizada em novembro de 2012. Os frutos foram colhidos manualmente pela manhã com pedúnculo e levados ao laboratório de Ciência e Tecnologia de Alimentos da Embrapa Hortaliças, onde foram selecionados de acordo com o tamanho, aparência comercial e sem danos aparente. $\mathrm{O}$ experimento foi conduzido em delineamento inteiramente casualizado, segundo um fatorial $6 \times 2$, com três repetições. Os tratamentos avaliados foram seis tempos de armazenamento $(0,3,6,9,12$ e 15 dias $)$ e dois tipos de embalagens poliméricas (policloreto de vinila - PVC; e polietileno tereftalato - PET) (Figura $1)$.

Os frutos foram acondicionados em bandeja de poliestireno envolvidas com filme de PVC esticável com 15 micras de espessura e, em embalagem do tipo PET $(19 \times 11 \times 4,8 \mathrm{~cm})$ perfurada (8 furos de aproximadamente $0,5 \mathrm{~cm}$ de diâmetro), contendo 30 frutos. Os frutos embalados foram mantidos a $10 \pm 1^{\circ} \mathrm{C}$ a $95 \pm 1 \%$ de umidade relativa. 


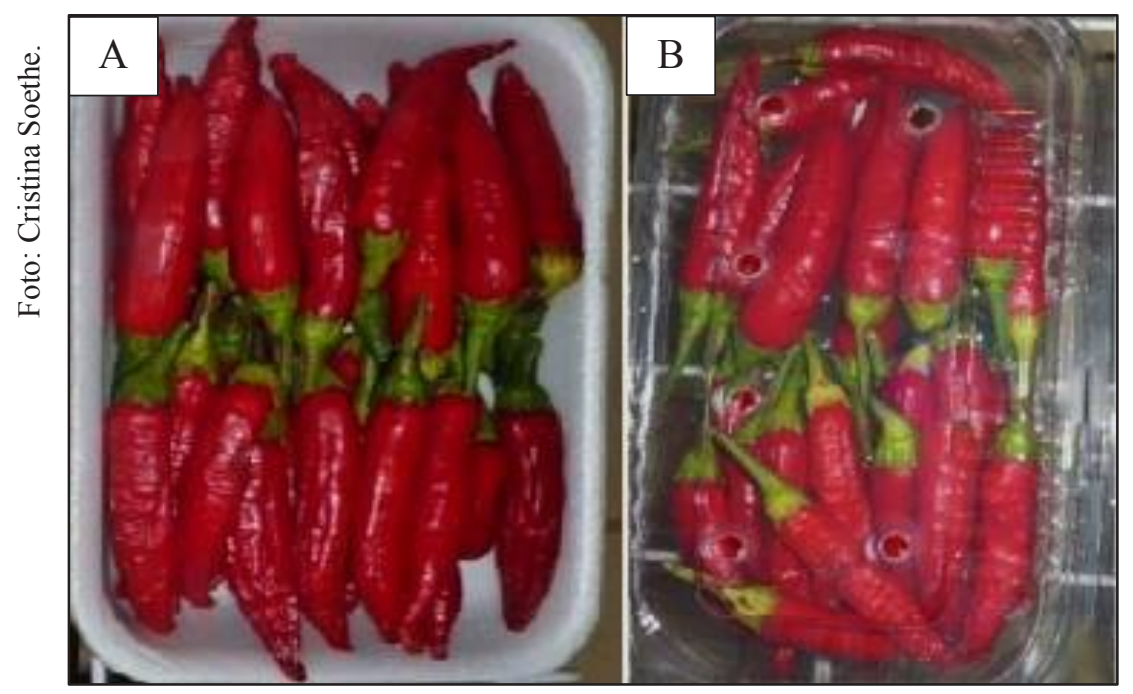

Figura 1. Pimentas dedo-de-moça 'BRS Mari' acondicionadas em bandeja de poliestireno envolta com filme de PVC (A) e embalagem PET (B) durante o armazenamento refrigerado.

Figure 1. "Dedo-de-moça" 'BRS Mari' pepper packed in polystyrene tray wrapped with PVC film (A) and PET packaging (B) during cold storage.

Após o armazenamento foram avaliados os seguintes parâmetros: sólidos solúveis (SS), acidez titulável (AT), coloração em termos de ângulo hue $\left(h^{\circ}\right)$, croma $(C)$ e luminosidade $\left(L^{*}\right)$, compostos fenólicos totais, atividade antioxidante e capsantina. As análises de SS, AT, cor e capsantina foram realizadas conforme descritas por MATTOS et al. (2007). A relação SS/AT foi determinada pela razão entre os atributos SS e AT.

A determinação do teor de compostos fenólicos totais foi realizada empregando o reagente de FolinCiocalteau, conforme descrita por ROESLER et al. (2007), com adaptações. Para a obtenção da curva padrão do ácido gálico utilizou-se concentrações de $0,10,30,50,70,90$ e 100 ppm. Para a obtenção dos extratos de pimenta, $5 \mathrm{~g}$ da amostra foram homogeneizados com $10 \mathrm{~mL}$ de metanol P.A. O homogenato foi submetido à agitação por 60 minutos e centrifugado por 5 minutos a $15000 \mathrm{rpm}$. Após filtração, o sobrenadante foi reservado para análise de compostos fenólicos. Para análise foram adicionados, 2,5 mL de Folin-Ciocalteau (1:3), 0,5 mL de amostra diluída (1:20) e 2,0 mL da solução de carbonato de sódio $10 \%$. Os tubos foram agitados, incubados por uma hora ao abrigo da luz e as amostras centrifugadas. Realizou-se a leitura no comprimento de onda de $765 \mathrm{~nm}$. Os resultados foram expressos em $\mathrm{mg}$ de equivalentes de ácido gálico por $100 \mathrm{~g}$ da amostra (mg EAG $100 \mathrm{~g}^{-1}$ ).

A determinação da atividade antioxidante foi baseada na extinção da absorção do radical 2,2-difenil-1-picril hidrazil (DPPH $60 \mu \mathrm{M}$ ) de acordo com RUFINO et al. (2007), com adaptações. Para a obtenção do extrato para quantificação da atividade antioxidante total, realizou-se o mesmo procedimento para obtenção do extrato dos compostos fenólicos. Foi adicionado $0,4 \mathrm{~mL}$ de cada extrato das amostras de pimenta diluído (1:20) e 1,6 mL do radical DPPH $(60 \mu \mathrm{M})$. Após 30 minutos de incubação, realizou-se leitura a $515 \mathrm{~nm}$ e foi convertida em porcentagem de Atividade Antioxidante (\% AA) utilizando a seguinte equação:

$$
\text { \%Inibição }=\left(\left(\mathrm{A}_{\text {controle }}-\mathrm{A}_{\text {Extrato }}\right) / \mathrm{A}_{\text {controle }}\right) * 100
$$

Para o fator tipo de embalagem as médias foram comparadas pelo teste $\mathrm{F}(\mathrm{p}<0,05)$ e para o fator tempo de armazenamento os dados foram submetidos à análise de variância e as médias comparadas pelo teste de Tukey $(\mathrm{p}<0,05)$. A variável atividade antioxidante foi submetida à análise de correlação de Pearson $(\mathrm{p}<0,01)$ com os teores de compostos fenólicos totais e de capsantina.

\section{RESULTADOS E DISCUSSÃO}

Não foi observado efeito da interação entre os fatores tipo de embalagem e tempo de armazenamento para todas as variáveis avaliadas (dados não apresentados). Quanto ao fator tipo de embalagem, não houve efeito para todas as variáveis avaliadas (Tabela 1). 
Tabela 1. Sólidos solúveis (SS; ${ }^{\circ}$ Brix), acidez titulável (AT; \% ácido cítrico), relação SS/AT, atributos de cor (luminosidade, $L^{*}$; croma, $C$; e ângulo $h u e, h^{o}$;), compostos fenólicos totais (CFT; mg EAG 100 $\mathrm{g}^{-1}$ ), atividade antioxidante (AA) e capsantina ( $\mathrm{mg} \mathrm{g}^{-1} \mathrm{MS}$ ) de pimentas dedo-de-moça 'BRS Mari' armazenadas sob refrigeração $\left(10 \pm 1^{\circ} \mathrm{C} / 95 \pm 1 \%\right.$ de UR) em dois tipos de embalagens poliméricas.

Table 1. Soluble solids (SS; ${ }^{\circ}$ Brix), titratable acidity (AT; \% citric acid), SS/AT ratio, color attributes (lightness, $L^{*}$; chroma, $C$; and hue angle, $\left.h^{\circ}\right)$, total phenolics compounds (CFT; $m g$ EAG $\left.100 \mathrm{~g}^{-1}\right)$, antioxidant activity (AA) and capsanthin ( $\left.g^{-1} \mathrm{mg} M S\right)$ of "dedo-de-moça" 'BRS Mari' pepper stored under refrigeration $\left(10 \pm 1{ }^{\circ} \mathrm{C} / 95 \pm 1 \%\right.$ UR) in two types of polymeric packaging.

\begin{tabular}{cccccccccc}
\hline \multirow{2}{*}{ Embalagem } & \multirow{2}{*}{ SS } & \multirow{2}{*}{ AT } & \multirow{2}{*}{ SS/AT } & \multicolumn{3}{c}{ Atributos de cor } & \multirow{2}{*}{ CFT } & AA & Capsantina \\
\cline { 5 - 6 } & & & & $L^{*}$ & $C$ & $h^{\circ}$ & & & \\
\hline PVC & $13,3^{\text {ns }}$ & $0,37^{\text {ns }}$ & $36,3^{\text {ns }}$ & $32,4^{\text {ns }}$ & $45,8^{\text {ns }}$ & $28,2^{\text {ns }}$ & $235,5^{\text {ns }}$ & $72,8^{\text {ns }}$ & $97,5^{\text {ns }}$ \\
PET & 13,2 & 0,37 & 35,8 & 32,6 & 45,7 & 28,1 & 238,6 & 72,8 & 95,6 \\
\hline CV (\%) & 8,14 & 3,63 & 8,22 & 2,53 & 1,60 & 1,87 & 2,67 & 5,39 & 11,94 \\
\hline
\end{tabular}

${ }^{\mathrm{ns}}$ diferenças não significativas pelo teste $\mathrm{F}(\mathrm{p}<0,05)$. $\mathrm{CV}=$ coeficiente de variação.

De acordo com BRACKMANN et al. (1999), filmes de PVC esticável de 15 micras de espessura apresentam baixa propriedade de barreira para $\mathrm{O}_{2}$ e $\mathrm{CO}_{2}$, não havendo intensa modificação da atmosfera, servindo apenas para evitar a desidratação dos frutos. A embalagem PET, por ser perfurada, não deve ter apresentado efeito sobre as pressões parciais de $\mathrm{O}_{2}$ e $\mathrm{CO}_{2}$, contudo, proporcionou, no interior da embalagem, a formação de um ambiente com alta umidade relativa, reduzindo a transpiração do fruto. Desta forma, o fato das embalagens testadas apresentarem reduzida capacidade de modificação atmosférica, em termos de redução de $\mathrm{O}_{2}$ e aumento de $\mathrm{CO}_{2}$, pode explicar a ausência de efeito da embalagem sobre as variáveis avaliadas.

Para o tempo de armazenamento não houve efeito para as variáveis SS, AT e relação SS/AT (Tabela 2), indicando que houve boa manutenção das características de sabor dos frutos. De acordo com MORGADO et al. (2008), o armazenamento refrigerado retarda modificações no teor de SS e AT. O valor da AT durante o armazenamento pode estar relacionado com o metabolismo respiratório, que tanto sintetiza quanto consome ácidos orgânicos (MORGADO et al. 2008).

Em relação à colheita, houve redução dos valores de $L^{*}$ no terceiro dia de armazenamento (Tabela 2), ocasionando perda da coloração vermelha brilhante, quando a partir desta avaliação pouca variação ocorreu até o final do experimento. LLANO et al. (2009) também observaram diminuição no valor de $L^{*}$ durante o armazenamento refrigerado de Capsicum annuum L.. De acordo com JHA \&
MATSUOKA (2002), a diminuição no brilho pode ser devido à perda de massa que afeta a reflectância, que é dependente da fonte e da intensidade de luz incidente.

Houve aumento do valor do ângulo hue e de croma nos frutos no terceiro dia de armazenamento refrigerado, quando a partir de então pouca alteração ocorreu até o $15^{\circ}$ dia de armazenamento (Tabela 2). O ângulo hue variou de 26,8 , na colheita, até 28,5 , maior valor observado a partir de seis dias de armazenamento, representando um incremento no valor do ângulo hue de aproximadamente 6,3\%. Embora tenha sido um aumento estatisticamente significativo, ambos os valores encontram-se na faixa do vermelho e é uma variação muito pequena, sendo imperceptível ao consumidor. $\mathrm{O}$ aumento no valor de croma indica aumento na intensidade da cor dos frutos. Segundo MENDONÇA et al. (2003), valores próximos de zero representam as cores neutras, enquanto que valores próximos de 60 expressam cores intensas. Neste trabalho, o valor de croma variou de 40,67 na colheita a 46,70 no $15^{\circ}$ dia de armazenamento. A intensidade da cor é fator importante, tendo em vista que a coloração vermelha é um dos atributos de qualidade destes frutos e de relevância para o consumidor.

O conteúdo de compostos fenólicos totais das pimentas diminuiu no terceiro dia de armazenamento, quando a partir desta avaliação pouca alteração ocorreu até o $15^{\circ}$ dia de armazenamento (Tabela 3). EDUSEI \& OFOSU-ANIM (2013) também observaram redução no teor de compostos fenólicos durante o armazenamento refrigerado de pimentão acondicionados em embalagens poliméricas. De acordo com YANG et al. (2011), durante o 
Tabela 2. Sólidos solúveis (SS), acidez titulável (AT; \% ácido cítrico), relação SS/AT e atributos de cor (luminosidade, $L^{*}$; croma, $C$; e ângulo $h u e, h^{\circ}$ ) de pimentas dedo-de-moça 'BRS Mari' em função do período de armazenamento refrigerado $\left(10 \pm 1^{\circ} \mathrm{C} / 95 \pm 1 \%\right.$ de UR).

Table 2. Soluble solids (SS), titratable acidity (AT; \% citric acid), SS/AT ratio and color attributes (lightness, $L^{*}$; chroma $C$; and hue angle $h^{\circ}$ ) of "dedo-de-moça" 'BRS Mari' pepper due to the cold storage period $\left(10 \pm 1^{\circ} \mathrm{C} / 95 \pm 1 \%\right.$ UR).

\begin{tabular}{ccccccc}
\hline \multirow{2}{*}{$\begin{array}{c}\text { Dias de } \\
\text { Armazenamento }\end{array}$} & $\begin{array}{c}\text { SS } \\
\left({ }^{\circ} \text { Brix }\right)\end{array}$ & AT & SS/AT & \multicolumn{3}{c}{ Atributos de cor } \\
\cline { 5 - 7 } & & & & $L^{*}$ & $C$ & $h^{\circ}$ \\
\hline 0 & $13,7^{\text {ns }}$ & $0,37^{\text {ns }}$ & $37,0^{\text {ns }}$ & $40,7 \mathrm{~b}$ & $26,8 \mathrm{~b}$ & $34,5 \mathrm{a}^{*}$ \\
3 & 13,4 & 0,36 & 37,4 & $47,2 \mathrm{a}$ & $28,0 \mathrm{a}$ & $32,0 \mathrm{~b}$ \\
6 & 13,1 & 0,37 & 35,2 & $46,4 \mathrm{a}$ & $28,5 \mathrm{a}$ & $32,0 \mathrm{~b}$ \\
9 & 13,8 & 0,39 & 36,6 & $46,3 \mathrm{a}$ & $28,5 \mathrm{a}$ & $32,4 \mathrm{~b}$ \\
12 & 12,9 & 0.36 & 35,4 & $47,3 \mathrm{a}$ & $28,4 \mathrm{a}$ & $32,3 \mathrm{~b}$ \\
15 & 12,6 & 0,36 & 34,8 & $46,7 \mathrm{a}$ & $28,5 \mathrm{a}$ & $32,0 \mathrm{~b}$ \\
\hline CV (\%) & 8,14 & 3,63 & 8,22 & 3,21 & 1,83 & 2,79 \\
\hline
\end{tabular}

${ }^{\bar{*}}$ Médias não seguidas pela mesma letra na coluna diferem pelo teste de Tukey $(\mathrm{p}<0,05)$.

${ }^{\text {ns }}$ diferenças não significativas pelo teste de Tukey $(\mathrm{p}<0,05)$. CV=coeficiente de variação.

Tabela 3. Teor de compostos fenólicos totais (CFT), atividade antioxidante (AA) e capsantina de pimentas dedo-de-moça 'BRS Mari' em função do período de armazenamento refrigerado $\left(10 \pm 1{ }^{\circ} \mathrm{C} / 95 \pm 1 \%\right.$ de UR).

Table 3. Content of total phenolic compounds (CFT), antioxidant activity (AA) and capsanthin of "dedo-demoça" 'BRS Mari' pepper due to the cold storage period ( $10 \pm 1{ }^{\circ} \mathrm{C} / 95 \pm 1 \%$ UR).

\begin{tabular}{cccc}
\hline Dias de Armazenamento & $\begin{array}{c}\text { CFT } \\
\left(\mathrm{mg} \mathrm{EAG} 100 \mathrm{~g}^{-1}\right)\end{array}$ & $\begin{array}{c}\text { AA } \\
(\%)\end{array}$ & $\begin{array}{c}\text { Capsantina } \\
\left(\mathrm{mg} \mathrm{g}^{-1} \mathrm{MS}\right)\end{array}$ \\
\hline 0 & $265,3 \mathrm{a}$ & $88,1 \mathrm{a}$ & $107,3^{\mathrm{ns}}$ \\
3 & $232,1 \mathrm{~b}$ & $67,7 \mathrm{~b}$ & 92,5 \\
6 & $231,3 \mathrm{~b}$ & $68,1 \mathrm{~b}$ & 88,6 \\
9 & $234,4 \mathrm{~b}$ & $71,3 \mathrm{~b}$ & 99,8 \\
12 & $227,1 \mathrm{~b}$ & $69,9 \mathrm{~b}$ & 96,3 \\
15 & $232,1 \mathrm{~b}$ & $71,6 \mathrm{~b}$ & 94,7 \\
\hline CV $(\%)$ & 3,96 & 7,72 & 11,94 \\
\hline
\end{tabular}

${ }^{*}$ Médias não seguidas pela mesma letra na coluna diferem pelo teste de Tukey $(\mathrm{p}<0,05)$.

${ }^{n s}$ diferenças não significativas pelo teste de Tukey $(p<0,05) . C V=$ coeficiente de variação. $E A G=$ equivalente de ácido gálico. $\mathrm{MS}=$ massa seca.

armazenamento dos frutos ocorre oxidação dos compostos fenólicos o que pode ocasionar redução da sua concentração na célula. Os compostos fenólicos e suas propriedades antioxidantes nos frutos podem ser modificados pelo ambiente e fatores pós-colheita, incluindo o armazenamento refrigerado, que promove oxidação enzimática e química destes compostos, contribuindo para a sua redução (KAUR \& KAPOOR 2001).

A atividade antioxidante das pimentas diminuiu no terceiro dia de armazenamento, sendo que após não foram observadas alterações significativas neste atributo (Tabela 3). VICENTE et al. (2005) também observaram redução na atividade antioxidante durante 
armazenamento a $10^{\circ} \mathrm{C}$ em frutos de Capsicum annuиm L. cv. Zafiro. De acordo com ROTILI et al. (2013), a atividade antioxidante em vegetais é decorrente da ação de uma variedade de compostos que são degradados ou sintetizados de acordo com o estado fisiológico e com os níveis de estresses abióticos e bióticos sofridos pelo fruto durante o armazenamento, o que pode resultar em redução da atividade antioxidante.

O conteúdo de capsantina das pimentas não foi influenciado durante o período de armazenamento (Tabela 3). A atmosfera modificada associada a refrigeração pode manter o teor de carotenoides em frutos (EDUSEI \& OFOSU-ANIM 2013). De acordo com SHI-LIN et al. (2013), a capsantina é o principal carotenoide em pimentas e responsável pela coloração vermelha, contribuindo com mais de 50\% dos carotenoides totais (PINTO et al. 2013).

Os valores de atividade antioxidante apresentaram correlação positiva e altamente significativa $(p<0,01)$ com o teor de compostos fenólicos, obtendo um coeficiente de correlação de 0,97 , sendo muito próximo de uma correlação linear perfeita. Correlação linear positiva entre atividade antioxidante e compostos fenólicos de pimentas tem sido relatada por outros autores (SAIDU \& GARBA 2011, CASTRO-CONCHA et al. 2014) e com outros compostos bioativos presentes em pimentas (BAE et al. 2012). De acordo com CUADRA-CRESPO \& DEL AMOR (2010), os compostos fenólicos podem atuar como antioxidantes, sugerindo que a atividade antioxidante presente em pimentas pode ser devido a presença de compostos fenólicos. Além disso, os compostos fenólicos são de grande importância na determinação de alguns atributos de qualidade em frutas e hortaliças, como cor, textura e sabor (CUADRA-CRESPO \& DEL AMOR 2010). De acordo com NADEEM et al. (2011), a capsantina é importante na eliminação dos radicais livres, podendo ser um dos fatores responsáveis pela atividade antioxidante em pimentas, porém, no presente trabalho, não houve correlação altamente significativa $(p<0,01)$ entre atividade antioxidante e o teor de capsantina. Considerando o efeito do tempo de armazenamento sobre a atividade antioxidante, os teores de compostos fenólicos e de capsantina e a análise de correlação é possível inferir que a redução da atividade antioxidante que ocorreu até o terceiro dia de armazenamento foi decorrente, principalmente, da redução do conteúdo dos compostos fenólicos.

\section{CONCLUSÃO}

Em pimentas dedo-de-moça 'BRS Mari' armazenadas sob refrigeração, não há diferença entre a embalagem bandejas de poliestireno revestidas com filme de policloreto de vinila (PVC) e embalagem de polietileno tereftalato (PET) perfurada para a manutenção da qualidade dos frutos.

O tempo de armazenamento refrigerado não interferiu na modificação dos teores de sólidos solúveis, acidez titulável e no conteúdo de capsantina, mas influenciou negativamente na aparência geral dos frutos, diminuiu o teor de compostos fenólicos e a atividade antioxidante até no terceiro dia de armazenamento refrigerado.

\section{REFERÊNCIAS}

BAE H et al. 2012. Variation of antioxidant activity and the levels of bioactive compounds in lipophilic and hydrophilic extracts from hot pepper (Capsicum spp.) cultivars. Food Chemistry 134: 1912-1918.

BRACKMANN A et al. 1999. Efeito de filmes de PVC esticável e polientileno no acúmulo de $\mathrm{CO}_{2}$ e na manutenção da qualidade pós-colheita de morangos cv. Tangi. Revista Brasileira de Agrociência 5: 89-92.

BRACKMANN A et al. 2008. Temperatura e umidade relativa na qualidade da tangerina 'Montenegrina' armazenada. Ciência Rural 38: 340-344.

CASTRO-CONCHA LA et al. 2014. Antioxidant capacity and total phenolic content in fruit tissues from accessions of Capsicum chinense Jacq. (Habanero Pepper) at different stages of ripening. The Scientific World Journal 2014: 1-5. CUADRA-CRESPO P \& DEL AMOR FM. 2010. Effects of postharvest treatments on fruit quality of sweet pepper at low temperature. Journal of the Science of Food and Agriculture 90: 2716-2722.

EDUSEI VO \& OFOSU-ANIM J. 2013. Biochemical changes in green chilli pepper fruits during storage in polymeric films. Journal of Research in Agriculture 2: 187192.

HENZ GP \& MORETTI CL. 2008. Colheita e PósColheita. In: RIBEIRO CSC et al. Pimentas Capsicum. Brasília: Embrapa Hortaliças. p.149-156.

JHA SN \& MATSUOKA T. 2002. Non-destructive techniques for quality evaluation of intact fruits and vegetables a review. Food Science and Technology Research 6: 284-285.

KAUR C \& KAPOOR HC. 2001. Antioxidants in fruits and vegetables the millennium's health. International Journal of Food Science and Technology 36: 703-725.

LLANO KRA et al. 2009. Quality and antioxidant properties of whole and fresh cut 'Cherry' peppers during storage at $10^{\circ} \mathrm{C}$. Facena $25: 21-32$. 
MANOLOPOULOU H et al. 2010. Modified atmosphere packaging storage of green bell peppers: quality criteria. Biosystems Engineering 106: 535-543.

MATTOS LM et al. 2007. Protocolos de avaliação da qualidade química e física de pimentas (Capsicum spp.). Brasília: Embrapa Hortaliças. 9p. (Comunicado Técnico $50)$.

MENDONÇA K et al. 2003. Concentração de etileno e tempo de exposição para desverdecimento de limão 'Siciliano'. Brazilian Journal of Food Technology 6: 179183.

MORGADO CMA et al. 2008. Conservação pós-colheita de frutos de pimentão sob diferentes condições de armazenamento e filmes. Horticultura Brasileira 26: 170174.

NADEEM M et al. 2011. Antioxidant Potential of Bell Pepper (Capsicum annum L.) a Review. Pakistan Journal of Food Sciences 21: 45-51.

NASSER ALM et al. 2005. Oligômeros em embalagem de pet para água mineral e suco de fruta. Uma revisão. Alimentos e Nutrição 16: 183-194.

PINTO CMF et al. 2013. Pimenta Capsicum: propriedades químicas, nutricionais, farmacológicas e medicinais e seu potencial para o agronegócio. Revista Brasileira de Agropecuária Sustentável 3: 108-120.

RODRIGUEZ-MATURINO A et al. 2012. Antioxidant activity and bioactive compounds of chiltepin (Capsicum annuum var. Glabriusculum) and habanero (Capsicum chinense): a comparative study. Journal of Medicinal Plants Research 6: 1758-1763.

ROESLER R et al. 2007. Atividade antioxidante de frutas do cerrado. Ciência e Tecnologia de Alimentos 27: 53-60.

ROTILI MCC et al. 2013. Composição, atividade antioxidante e qualidade do maracujá-amarelo durante armazenamento. Semina: Ciências Agrárias 34: 227-240.

RUFINO MSM et al. 2007. Metodologia científica: determinação da atividade antioxidante total em frutas pela captura do radical livre DPPH. Fortaleza: Embrapa Agroindústria Tropical. 4p. (Comunicado Técnico 127).

SAIDU AN \& GARBA R. 2011. Antioxidant activity and phytochemical screening of five species of Capsicum fruits. International Research Journal of Biochemistry and Bioinformatics 1: 237-241.

SARANTÓPOULOS CIGL et al. 2003. Efeitos da embalagem e da temperatura de estocagem na qualidade de couve minimamente processada. Brazilian Journal of Food Technology 6: 185-190.

SHI-LIN T et al. 2013. Effects of fruit bagging on capsanthin and expression of key genes in the capsanthin biosynthetic pathway during fruit development and ripening of the pepper. Research Journal of Biotechnology 8: 118-123.

TOPUZ A et al. 2011. Influence of different drying methods on carotenoids and capsaicinoids of paprika ( $\mathrm{Cv}$., Jalapeno). Food Chemistry 129: 860-865.

UTTO W et al. 2013. Influences of modified atmosphere packaging using active breathable films on antioxidant activity and quality of minimally processed Teaw leaves (Cratoxylum formosum Dyer). International Food Research Journal 20: 661-671.

VERA-GUZMÁN AM et al. 2011. Phytochemical evaluation of wild and cultivated pepper (Capsicum annuum L. and C. pubescens Ruiz \& Pav.) from Oaxaca, Mexico. Chilean Journal of Agricultural Research 71: 578585.

VICENTE AR et al. 2005. UV-C treatments reduce decay, retain quality and alleviate chilling injury in pepper. Postharvest Biology and Technology 35: 69-78.

YANG Y et al. 2011. Identification of phenolics in Chinese toon and analysis of their content changes during storage. Food Chemistry 128: 831-838. 